\title{
Association of abnormal glucose metabolism and insulin resistance in patients with atypical and typical endometrial cancer
}

\author{
YONGJING LAI and CHUANYING SUN \\ Department of Obstetrics and Gynecology, People's Hospital of Rizhao, Rizhao, Shandong 276800, P.R. China
}

Received August 11, 2017; Accepted November 29, 2017

DOI: 10.3892/ol.2017.7590

\begin{abstract}
This study aimed to detect glucose metabolism indicators and insulin resistance index in patients with endometrial cancer, and to explore the clinical significance and correlation between them. A total of 65 patients with endometrial cancer (52 of typical endometrial and 13 cases of atypical endometrial cancer patients, 27 with diabetes mellitus, and 38 cases without diabetes mellitus) were selected at the People's Hospital of Rizhao from June, 2010 to June, 2016 to serve as the observation group. During the same period, 62 patients with endometrial benign lesions ( 24 with diabetes mellitus and 38 cases without diabetes mellitus) were selected as the control group. General information including height, body weight, body mass index (BMI), abdominal, waist and hip circumference, and waist-to-hip ratio (WHR) was compared between the two groups. Fasting blood glucose, glycosylated hemoglobin, fasting insulin level (FINS), insulin resistance index (HOMA-IR), follicle estrogen (FSH), luteinizing hormone and estradiol (estrogen) were detected and compared between the two groups. Multivariate logistic regression was used to analyze the risk factors for endometrial cancer. The results showed that there were no significant differences in the height and hip circumference among the typical, atypical and control groups. By contrast, weight, BMI, waist circumference, abdominal circumference and the WHR of the typical group were significantly higher than those of the atypical and control groups $(\mathrm{P}<0.05)$. No significant differences were found between the atypical and control groups $(\mathrm{P}>0.05)$. Levels of the FINS and HOMA-IR typical group were significantly higher than those in the atypical and control groups, and the incidence of hyperinsulinemia and insulin resistance was significantly higher in the observation than in the control group $(\mathrm{P}<0.05)$. Of the patients with diabetes, the levels of FINS, HOMA-IR and estrogen were significantly higher, but the level of FSH was
\end{abstract}

Correspondence to: Dr Yongjing Lai, Department of Obstetrics and Gynecology, People's Hospital of Rizhao, 126 Taian Road, Rizhao, Shandong 276800, P.R. China

E-mail: yongjing_lai@163.com

Key words: diabetes mellitus, typical endometrial cancer, atypical endometrial cancer, insulin resistance significantly lower in the observation compared to the control group $(\mathrm{P}<0.05)$. For patients without diabetes, significant differences in the levels of FINS and HOMA-IR were found between the observation and control groups $(\mathrm{P}<0.05)$. There was no significant difference in the levels of FINS and HOMA-IR among endometrial cancer patients with different pathological features $(\mathrm{P}>0.05)$. HOMA-IR $(\mathrm{OR}=1.240)$, estrogen $(\mathrm{OR}=1.192)$ and $\mathrm{FSH}(\mathrm{OR}=1.002)$ are risk factors for endometrial cancer. The results suggest that hyperinsulinemia and insulin resistance are risk factors of endometrial cancer. Insulin may therefore be involved in the development of endometrial cancer by affecting the level of sex hormones.

\section{Introduction}

Endometrial cancer is a type of female malignant tumor with the highest incidence of all female cancers in developed countries (1). At present, the etiology of endometrial cancer remains unclear. The theory of 'estrogen without progesterone' (decrease in the level of progesterone before menopause that cannot antagonize estrogen, and an increase in the level of estrogen after menopause) posits that the occurrence of endometrial cancer is correlated with the disorder of estrogen and progesterone (2).

Clinical studies found that the incidence of endometrial cancer significantly increased after menopause without an increase in estrogen levels, indicating the existence of an estrogen-independent mechanism for the occurrence of endometrial cancer (3). The three major risk factors for endometrial cancer include obesity, diabetes and hypertension. All three factors share the common pathophysiological basis of insulin resistance and hyperinsulinemia (4). Insulin resistance and hyperinsulinemia can increase the risk of multiple malignancies, especially colorectal and endometrial cancer (5). Previous findings showed that the risk of endometrial cancer in patients with diabetes is double that of the normal population (6). Therefore, monitoring of indicators of glucose metabolism and insulin resistance index is of great significance for the assessment of the risk of endometrial cancer. The association between endometrial cancer and insulin resistance has become a focus of the studies on endometrial cancer. To the best of our knowledge, studies on the comparison of estrogen-dependent (typical) and estrogen-independent (atypical) endometrial cancer are relatively rare. 
In the present study, the association between abnormal glucose metabolism and insulin resistance in patients with atypical and typical endometrial cancer was analyzed to explore the clinical significance of abnormal glucose metabolism and insulin resistance in early diagnosis, early treatment and the prevention of endometrial cancer.

\section{Materials and methods}

Clinical data. A total of 65 endometrial cancer patients with complete clinical data were selected at the People's Hospital of Rizhao (Shandong, China) from June, 2010 to June, 2016. The patients were diagnosed by pathological examination. Inclusion criteria were: Patients with only primary tumor and without other malignancies, and patients who received no other treatments prior to surgery. The average age in the observation group was $56.3 \pm 14.2$ years. Typical endometrial cancer (endometrioid adenocarcinoma) was observed in 52 cases and atypical endometrial cancer was observed in 13 cases; (6, adenosquamous carcinoma; 2, of mucinous adenocarcinoma; 2, of serous adenocarcinoma; 2 , of clear cell carcinoma; and 1 case of undifferentiated carcinoma); 27, of diabetes; 38, showed no diabetes; 43, of menopause; and 22, of non-menopause. During the same period, 62 patients with endometrial benign lesions were selected as the control group. Patients with a history of cancer and the use of steroids were excluded. The average age of the control group was 57.1 \pm 13.7 years, and included 24 cases with diabetes, 38 without diabetes, 41 of menopause, and 21 of non-menopause. No significant differences in the average age, diabetes prevalence and proportion of menopause were found between groups $(\mathrm{P}>0.05)$.

This study was approved by the Ethics Committee of People's Hospital of Rizhao. All the patients signed informed consent.

Research methods. General clinical information including age, menstrual, marriage, disease (polycystic ovary syndrome, diabetes and hypertension) and family history, surgical methods, type of pathology, histological grade, estrogen receptor (ER) positive/negative, and progesterone receptor (PR) positive/negative were recorded.

The weight of fasted patients coated with single layer clothes was measured. Waist, abdominal and hip circumference were measured under smooth breathing. Shoes and hats were removed to measure height. All the indicators were measured twice to calculate the average value. Body mass index (BMI) was calculated as height/weight ${ }^{2} ; 24 \leq \mathrm{BMI}<28$ represents overweight, and BMI $\geq 28$ represents obesity. The waist-to-hip ratio (WHR) was calculated as waist/hip circumference.

Detection of serum markers. Venous blood (3-5 ml) was extracted through elbow vein from fasted patients in the morning, and transferred to an anticoagulant tube, followed by centrifugation at $3,000 \mathrm{~g}$ for $15 \mathrm{~min}$ to collect serum. Fasting blood glucose (FBG) and glycosylated hemoglobin (HbA1c) were detected using the glucose-oxidase method. Level of fasting insulin (FINS) was measured by electrochemiluminescence method. A level of FINS higher than the level of the control group + upper limit of $95 \%$ CI indicated an increase of FINS (hyperinsulinemia). Levels of follicle-stimulating hormone (FSH), luteinizing hormone ( $\mathrm{LH})$ and estrogen were measured by radioimmunoassay. Insulin resistance was assessed using homeostasis model assessment (HOMA) (7). Insulin resistance index (HOMA-IR) was calculated as (FPG mmol/1 x FINS mU/l)/22.5 with values higher than that of control group HOMA-IR indicating insulin resistance (8).

Statistical analysis. SPSS 20.0 software (IBM Corp., Armonk, NY,USA) was used for all the statistical analyses. Measurement data were expressed as mean $\pm \mathrm{SD}$, and comparison between groups were performed using t-test. Countable data were expressed as a percentage, and comparisons between groups were performed using the Fisher's exact test or Chi-square test. Logistic regression was performed for multi-factor analysis. $\mathrm{P}<0.05$ was considered to indicate a statistically significant difference.

\section{Results}

Comparison of general information between the observation group (typical and atypical) and control groups. Significant differences in weight, BMI, waist circumference, abdominal circumference and WHR $(\mathrm{P}<0.05)$, but not in height and hip circumference were found among the typical, atypical and control groups. The weight, BMI, waist and abdominal circumference were significantly higher in the typical than in the atypical and control groups. By contrast, no significant differences in those indicators were found between the atypical and control groups (Table I).

Comparison of glucose metabolic index and sex hormone level between the observation (typical and atypical) and control groups. Significant differences in the levels of FINS, HOMA-IR, FSH and estrogen, but not in the levels of FBG, $\mathrm{HbAlc}$ and $\mathrm{LH}$ were found among the typical, atypical and control groups. Levels of FINS and HOMA-IR in the typical group were significantly higher than those in the atypical and control groups $(\mathrm{P}<0.05)$. However, no significant differences were found between the atypical and control groups $(\mathrm{P}>0.05)$. A significantly higher level of FSH and lower level of estrogen were found in the typical and atypical groups than in the control group $(\mathrm{P}<0.05)$. With the level of FINS $>15.32 \mathrm{pmmol} / \mathrm{l}$ (level of control group $+95 \% \mathrm{CI}$ upper limit) as a diagnostic criterion, increased FINS was found in 46 cases of the observation group, accounting for $70.77 \%(46 / 65)$, and 5 cases in the control group, accounting for $8.06 \%(5 / 62)$, with significant differences found between them $(\mathrm{P}<0.05)$. With HOMA-IR $>2.29$ as the diagnostic criterion (value of control group), insulin resistance was found in 52 cases of the observation group, accounting for $80.0 \%$ (52/65), and in 8 cases of the control group, accounting for $12.90 \%$, with significant differences found between them $(\mathrm{P}<0.05)$ (Tables II and III).

Comparison of glucose metabolic indexes between the diabetes and non-diabetes groups. Significant differences in the levels of FINS and HOMA-IR $(\mathrm{P}<0.05)$, but not in the levels of FBG and HbA1c ( $>>0.05)$, were found between the the observation and control groups in patients with or without diabetes (Table IV). 
Table I. Comparison of general information among the typical, atypical and control groups (mean \pm SD).

\begin{tabular}{|c|c|c|c|c|c|}
\hline \multirow[b]{2}{*}{ Items } & \multicolumn{3}{|c|}{ Groups } & \multirow[b]{2}{*}{$\mathrm{t} / \chi^{2}$ value } & \multirow[b]{2}{*}{ P-value } \\
\hline & Typical $(\mathrm{n}=52)$ & Atypical $(n=13)$ & Control $(n=62)$ & & \\
\hline Height (m) & $1.61 \pm 0.04$ & $1.60 \pm 0.05$ & $1.60 \pm 0.03$ & 0.358 & 0.714 \\
\hline Weight (kg) & $70.22 \pm 8.17$ & $63.14 \pm 9.32^{\mathrm{a}}$ & $62.76 \pm 8.53^{a}$ & 5.865 & 0.003 \\
\hline BMI $\left(\mathrm{kg} / \mathrm{m}^{2}\right)$ & $27.58 \pm 2.64$ & $24.23 \pm 2.97^{\mathrm{a}}$ & $24.18 \pm 3.21^{\mathrm{a}}$ & 7.697 & 0.002 \\
\hline Waist circumference $(\mathrm{cm})$ & $98.03 \pm 7.89$ & $92.06 \pm 9.24^{\mathrm{a}}$ & $91.69 \pm 10.30^{\mathrm{a}}$ & 3.932 & 0.009 \\
\hline Abdominal circumference $(\mathrm{cm})$ & $102.78 \pm 5.46$ & $96.18 \pm 4.68^{a}$ & $95.98 \pm 5.17^{\mathrm{a}}$ & 3.251 & 0.032 \\
\hline Hip circumference $(\mathrm{cm})$ & $99.04 \pm 5.87$ & $96.14 \pm 5.28$ & $96.02 \pm 5.62$ & 1.423 & 0.316 \\
\hline WHR & $0.984 \pm 0.05$ & $0.945 \pm 0.04^{\mathrm{a}}$ & $0.942 \pm 0.06^{\mathrm{a}}$ & 4.684 & 0.007 \\
\hline
\end{tabular}

${ }^{\mathrm{a} C}$ Compared with typical group, $\mathrm{P}<0.05$; BMI, body mass index; WHR, waist-to-hip ratio; SD, standard deviation.

Table II. Comparison of glucose metabolic index and sex hormone level among the typical, atypical and control groups $($ mean $\pm \mathrm{SD})$.

\begin{tabular}{|c|c|c|c|c|c|}
\hline \multirow[b]{2}{*}{ Items } & \multicolumn{3}{|c|}{ Groups } & \multirow[b]{2}{*}{$\mathrm{t} / \chi^{2}$ value } & \multirow[b]{2}{*}{ P-value } \\
\hline & Typical $(n=52)$ & Atypical $(n=13)$ & Control $(n=62)$ & & \\
\hline $\mathrm{FBG}(\mathrm{mmol} / \mathrm{l})$ & $5.21 \pm 0.34$ & $5.15 \pm 0.33$ & $5.13 \pm 0.35$ & 0.485 & 0.641 \\
\hline $\mathrm{HbA1c}(\%)$ & $5.87 \pm 0.56$ & $5.68 \pm 0.51$ & $5.64 \pm 0.49$ & 0.865 & 0.073 \\
\hline FINS (pmmol/l) & $18.45 \pm 5.32$ & $10.23 \pm 3.47^{\mathrm{a}}$ & $10.18 \pm 3.21^{\mathrm{a}}$ & 7.967 & 0.003 \\
\hline HOMA-IR & $3.93 \pm 1.19$ & $2.36 \pm 1.06^{\mathrm{a}}$ & $2.29 \pm 1.02^{\mathrm{a}}$ & 3.932 & 0.008 \\
\hline FSH (mIU/ml) & $37.84 \pm 9.65$ & $36.47 \pm 9.65$ & $50.21 \pm 9.76^{\mathrm{a}, \mathrm{b}}$ & 8.215 & 0.002 \\
\hline LH (mIU/ml) & $29.12 \pm 10.07$ & $29.65 \pm 10.28$ & $30.13 \pm 10.62$ & 0.634 & 0.516 \\
\hline $\mathrm{E} 2(\mathrm{pg} / \mathrm{ml})$ & $472.32 \pm 56.25$ & $462.32 \pm 55.76$ & $367.61 \pm 55.43^{\mathrm{a}, \mathrm{b}}$ & 4.864 & 0.006 \\
\hline
\end{tabular}

${ }^{\mathrm{a} C o m p a r e d}$ with typical group, $\mathrm{P}<0.05$; ${ }^{\mathrm{b}}$ compared with atypical group, $\mathrm{P}<0.05$; FBG, fasting blood glucose glucose; HbA1c, glycosylated hemoglobin; FINS, fasting insulin level; HOMA-IR, insulin resistance index; FSH, follicle estrogen; LH, luteinizing hormone; SD, standard deviation.

Table III. Comparison of incidence of hyperinsulinemia and insulin resistance between observation group and control group (cases, \%).

\begin{tabular}{lrrrr}
\hline \multicolumn{4}{c}{ Groups } & \\
Items & Observation & \multicolumn{1}{c}{ Control } & \multirow{2}{*}{$\chi^{2}$ value } & P-value \\
\cline { 2 - 4 } FINS & $70.77(46 / 65)$ & $8.06(5 / 62)$ & 7.653 & 0.001 \\
HOMA-IR & $80.0(52 / 65)$ & $12.90 \%(8 / 62)$ & 5.346 & 0.002 \\
\hline
\end{tabular}

FINS, fasting insulin level; HOMA-IR, insulin resistance index.

Comparison of levels of FINS and HOMA-IR in patients with different pathological features. No significant differences in the levels of FINS and HOMA-IR were found in patients with different pathological features (histopathological grade, FIGO stage, and ER and PR expression) ( $\mathrm{P}>0.05$ ) (Table V).

Multivariate logistic regression analysis. Multivariate logistic regression analysis revealed that HOMA-IR
$(\mathrm{OR}=1.283)$, FINS $(\mathrm{OR}=1.240)$, estrogen $(\mathrm{OR}=1.192)$ and $\mathrm{FSH}(\mathrm{OR}=1.002)$ were the risk factors for endometrial cancer. HOMA-IR showed the strongest correlation with endometrial carcinoma, followed by FINS, estrogen and FSH. A lower level of FSH indicated, a higher risk of endometrial cancer (Table VI).

\section{Discussion}

According to different pathogenesis, Bristow believed that endometrial cancer can be divided into the estrogen-dependent and -independent clinicopathological types (9).

Type I is estrogen-dependent endometrial cancer, and the occurrence of type I is likely caused by the effects of estrogen with the antagonist of progesterone in endometrium. Type I accounts for $80-90 \%$ of endometrial cancer, and the histopathologic types are adenocarcinoma. Type I mainly affects young patients, especially ones combined with metabolic syndrome (obesity, hypertension, diabetes), infertility, delayed menopause, and a family history of endometrial cancer. Patients with type I endometrial cancer usually present as PR-positive and have a favorable prognosis (10). In the present study, type I 
Table IV. Comparison of glucose metabolic indexes between diabetes and non-diabetes group (mean $\pm \mathrm{SD})$.

\begin{tabular}{|c|c|c|c|c|}
\hline \multirow[b]{3}{*}{ Items } & \multicolumn{4}{|c|}{ Groups } \\
\hline & \multicolumn{2}{|c|}{ Diabetes } & \multicolumn{2}{|c|}{ Non-diabetes } \\
\hline & Observation $(n=27)$ & Control $(n=24)$ & Observation $(\mathrm{n}=38)$ & Control $(n=38)$ \\
\hline FBG (mmol/l) & $5.56 \pm 1.51$ & $5.53 \pm 1.43$ & $4.95 \pm 1.12$ & $4.87 \pm 1.09$ \\
\hline $\operatorname{HbA} 1 \mathrm{c}(\%)$ & $6.39 \pm 0.46$ & $6.33 \pm 0.52$ & $5.76 \pm 0.57$ & $5.71 \pm 0.53$ \\
\hline FINS (pmmol/l) & $21.05 \pm 5.47$ & $12.93 \pm 4.86^{\mathrm{a}}$ & $17.02 \pm 6.54$ & $9.13 \pm 3.62^{\mathrm{a}}$ \\
\hline HOMA-IR & $4.48 \pm 1.12$ & $2.89 \pm 1.03^{\mathrm{a}}$ & $3.45 \pm 1.13$ & $1.98 \pm 0.92^{\mathrm{a}}$ \\
\hline
\end{tabular}

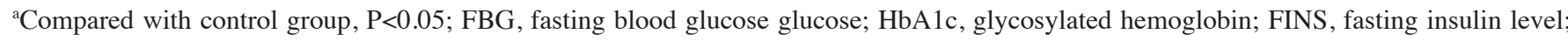
HOMA-IR, insulin resistance index; SD, standard deviation.

Table V. Comparison of levels of FINS and HOMA-IR among patients with different pathological features (mean \pm SD).

\begin{tabular}{|c|c|c|c|c|c|c|c|}
\hline Pathological features & $\mathrm{n}$ & FINS (pmmol/l) & $\chi^{2}$ value & P-value & HOMA-IR & $\chi^{2}$ value & P-value \\
\hline Pathological grades & & & 2.65 & 0.094 & & 2.17 & 0.011 \\
\hline $\mathrm{G} 1$ & 23 & $20.01 \pm 6.52$ & & & $3.93 \pm 1.79$ & & \\
\hline $\mathrm{G} 2$ & 28 & $17.32 \pm 6.43$ & & & $3.82 \pm 1.85$ & & \\
\hline G3 & 14 & $18.24 \pm 5.96$ & & & $3.74 \pm 1.91$ & & \\
\hline FIGO stage & & & 5.87 & 0.059 & & 4.78 & 0.062 \\
\hline I-II & 56 & $17.85 \pm 6.14$ & & & $3.83 \pm 1.87$ & & \\
\hline III-IV & 9 & $13.26 \pm 5.98$ & & & $3.54 \pm 1.96$ & & \\
\hline PR & & & 3.54 & 0.083 & & 3.87 & 0.078 \\
\hline Positive & 52 & $18.61 \pm 5.96$ & & & $3.84 \pm 1.58$ & & \\
\hline Negative & 13 & $16.23 \pm 6.05$ & & & $3.69 \pm 1.84$ & & \\
\hline ER & & & 4.12 & 0.075 & & 4.32 & 0.069 \\
\hline Positvie & 51 & $18.78 \pm 6.27$ & & & $3.85 \pm 1.82$ & & \\
\hline Negative & 14 & $16.06 \pm 5.83$ & & & $3.66 \pm 1.70$ & & \\
\hline
\end{tabular}

FIGO, International Federation of Gynecology and Obstetrics; PR, progesterone receptor; ER, estrogen receptor; SD, standard deviation.

Table VI. Multivariate logistic regression analysis.

\begin{tabular}{|c|c|c|c|c|}
\hline Factors & b value & Wald $\chi^{2}$ value & P-value & OR $(95 \%$ confidence interval) \\
\hline FINS & 0.775 & 7.324 & 0.003 & $1.240(1.085-1.461)$ \\
\hline HOMA-IR & 0.386 & 8.642 & 0.000 & $1.283(1.096-1.567)$ \\
\hline FSH & -0.674 & 5.813 & 0.025 & $1.002(0.942-1.012)$ \\
\hline $\mathrm{E} 2$ & 0.873 & 6.701 & 0.013 & $1.192(1.083-1.386)$ \\
\hline
\end{tabular}

FINS, fasting insulin level; HOMA-IR, insulin resistance index; FSH, follicle estrogen.

endometrial cancer accounted for $80.0 \%(52 / 65)$ of all the cases, which is consistent with previous studies (11). Type II is estrogen-independent endometrial cancer. In type II, atrophic endometrium can be observed around lesions. Occurrence of this type shows no correlation with estrogen, but is closely correlated with mutations in p53, inactivation of E-cadherin and overexpression of Her2/neu (12). Type II is relatively rare, and histopathological types mainly include mucinous adenocarcinoma, serous adenocarcinoma, clear cell carcinoma and other non-uterine-like adenocarcinoma. Type II endometrial cancer mainly affects elderly thin women. Tumor differentiation is usually poor and PR is usually negative. Patients with type II endometrial cancer usually show a high degree of malignancy and poor prognosis (13). 
Previous findings have shown that obesity is the primary risk factor for endometrial cancer, and weight 13.5 and $22.5 \mathrm{~kg}$ higher than the ideal value can increase the risk of endometrial cancer by 3 - and 10- fold, respectively (14).

BMI is another indicator of obesity. Findings have shown that patients with a higher BMI usually have a higher risk of endometrial cancer (15). In the present study, WHR was significantly higher in the typical than in the control group $(\mathrm{P}<0.05)$, which is consistent with previous findings showing that patients with upper body obesity have a higher risk of endometrial cancer compared to patients with other types of obesity (16). Type 2 diabetes is another risk factor for endometrial cancer. It has been shown that, serum insulin levels can increase the incidence of endometrial cancer in a dose-dependent manner. Additionally, hyperinsulinemia is considered to be an independent risk factor for the incidence of endometrial cancer (17). Type 2 diabetes, not only increases the incidence of EC (18), but also increases the relative risk of death in EC patients (19). Type 2 diabetes combined with obesity can significantly increase the risk of EC $(20,21)$. In the present study, the levels of FINS and HOMA-IR were significantly higher in the typical group than in the atypical and control groups $(\mathrm{P}<0.05)$. The incidence of hyperinsulinemia and insulin resistance was also significantly higher in the observation group than that in the control group $(\mathrm{P}<0.05)$. Insulin resistance is not a disease, but the key pathophysiological process of obesity, diabetes, hypertension and even metabolic syndrome. Poor lifestyle is the leading cause of obesity, diabetes and other insulin resistance. Modified diet structure and appropriate physical exercise is imperative in preventing obesity, diabetes and other diseases, but can also effectively prevent endometrial cancer.

Insulin resistance is defined as the reduced biological effect of a certain amount of insulin after binding to the receptor. Insulin resistance is manifested by the decreased use and increased output of peripheral glucose (22). At the early stage of insulin resistance, pancreatic $\beta$-cells will secrete excessive insulin to induce hyperinsulinemia. With the exhaustion of $\beta$-cells, blood glucose levels continue to rise, ultimately leading to the development of diabetes (23). In the present study, the incidence of hyperinsulinemia in the observation group was $70.77 \%$, which is significantly higher than that of the control group $(8.06 \%)$. According to the HOMA-IR evaluation method, $80.0 \%$ of patients in the observation group had insulin resistance, which was significantly higher than that of the control group. With the improvement of insulin resistance or metabolic abnormalities, the risk of endometrial cancer can also be reduced (24). However, the mechanism of the role of insulin in endometrial cancer remains unclear.

The possible mechanisms involved the fact that, insulin can bind to insulin or vascular endothelial growth factor receptor in endometrial cells to promote cell proliferation, inhibit apoptosis, induce the expression of vascular endothelial growth factor, and promote angiogenesis, which in turn leads to the occurrence of endometrial cancer. Additionally insulin is involved in tumor development by directly or indirectly affecting endogenous estrogen metabolism (25). Under physiological conditions, $30-50 \%$ of estrogen in the plasma can bind to the sex hormone-binding globulin (SHBG) to form an inactive form, while high insulin can inhibit the secretion of SHBG to increase free active estrogen in blood circulation (26). In addition, high insulin can affect the expression of adipose tissue aromatase P450c17 in ovarian and adrenal gland, promote androgen synthesis, and increase estrogen peripheral transformation (27). Insulin can also promote the expression of endometrial ER, which in turn enhances the function of estrogen (28). Insulin can continue to the proliferation of endometrial cells under the effects of estrogen, thereby increasing the incidence of endometrial cancer (29).

The results of the present study have shown that HOMA-IR $(\mathrm{OR}=1.283)$, FINS $(\mathrm{OR}=1.240)$, estrogen $(\mathrm{OR}=1.192)$ and $\mathrm{FSH}$ $(\mathrm{OR}=1.002)$ were risk factors for endometrial cancer. Of these, HOMA-IR was strongly correlated with endometrial carcinoma, followed by FINS, estrogen and FSH. Higher levels of HOMA-IR, FINS and estrogen, and lower levels of FSH can increase the risk of endometrial cancer. Insulin may also be involved in the development of endometrium by affecting the levels of sex hormone. Multi-center studies with large sample size should be performed to detect the abovementioned indicators to block high-risk factors of endometrial cancer (30), in order to achieve the early prevention and treatment of endometrial cancer.

\section{References}

1. Jemal A, Siegel R, Ward E, Murray T, Xu J, Smigal C and Thun MJ: Cancer statistics, 2006. CA Cancer J Clin 56: 106-130, 2006.

2. Bokhman JV: Two pathogenetic types of endometrial carcinoma. Gynecol Oncol 15: 10-17, 1983.

3. Bodner-Adler B, Bodner K, Obermair A, Czerwenka K, Petru E, Leodolter S and Mayerhofer K: Prognostic parameters in carcinosarcomas of the uterus: A clinico-pathologic study. Anticancer Res 21: 3069-3074, 2001.

4. Sanz-Chávez TL, Vilar-Compte D, de Nicola-Delfín L and Meneses-García A: Overweight, obesity, diabetes, and hypertension in endometrial cancer. Rev Med Inst Mex Seguro Soc 51: 326-329, 2013 (In Spanish)

5. Hursting SD and Berger NA: Energy balance, host-related factors, and cancer progression. J Clin Oncol 28: 4058-4065, 2010.

6. Liu Y, Tian JW, Xu Y and Cheng W: Role of transvaginal contrast-enhanced ultrasound in the early diagnosis of endometrial carcinoma. Chin Med J 125: 416-421, 2012.

7. Matthews DR, Hosker JP, Rudenski AS, Naylor BA, Treacher DF and Turner RC: Homeostasis model assessment: Insulin resistance and beta-cell function from fasting plasma glucose and insulin concentrations in man. Diabetologia 28: 412-419, 1985.

8. Chen WJ, Liu Y, Sui YB, Zhang B, Zhang XH and Yin XH: Increased circulating levels of musclin in newly diagnosed type 2 diabetic patients. Diab Vasc Dis Res 14: 116-121, 2017.

9. Bristow RE: Endometrial cancer. Curr Opin Oncol 11: 388-393, 1999.

10. Clement PB and Young RH: Endometrioid carcinoma of the uterine corpus: A review of its pathology with emphasis on recent advances and problematic aspects. Adv Anat Pathol 9: 145-184, 2002.

11. Lukanova A, Lundin E, Micheli A, Arslan A, Ferrari P, Rinaldi S Krogh V, Lenner P, Shore RE, Biessy C, et al: Circulating levels of sex steroid hormones and risk of endometrial cancer in postmenopausal women. Int J Cancer 108: 425-432, 2004.

12. Lax SF, Pizer ES, Ronnett BM and Kurman RJ: Clear cell carcinoma of the endometrium is characterized by a distinctive profile of p53, Ki-67, estrogen, and progesterone receptor expression. Hum Pathol 29: 551-558, 1998.

13. Singh M, Darcy KM, Brady WE, Clubwala R, Weber Z, Rittenbach JV, Akalin A, Whitney CW, Zaino R, Ramirez NC, et al: Cadherins, catenins and cell cycle regulators: Impact on survival in a gynecologic oncology group phase II endometrial cancer trial. Gynecol Oncol 123: 320-328, 2011. 
14. Sato E, Nakayama K, Nakamura K, Ishikawa N, Ishikawa M, Minamoto T, Ishibashi T and Kyo S: Efficacy of metformin for advanced-stage endometrial cancer: A case report. Mol Clin Oncol 6: 441-443, 2017

15. Trentham-Dietz A, Nichols HB, Hampton JM and Newcomb PA: Weight change and risk of endometrial cancer. Int J Epidemiol 35 $151-158,2006$

16. Xu WH, Matthews CE, Xiang YB, Zheng W, Ruan ZX, Cheng JR, Gao YT and Shu XO: Effect of adiposity and fat distribution on endometrial cancer risk in Shanghai women. Am J Epidemiol 161: 939-947, 2005.

17. Mu N, Zhu Y, Wang Y, Zhang H and Xue F: Insulin resistance: A significant risk factor of endometrial cancer. Gynecol Oncol 125: 751-757, 2012.

18. Kacalska O, Krzyczkowska-Sendrakowska M, Milewicz T, Zabińska-Popiela M, Bereza T, Krzysiek-Maczka G and Krzysiek J: Molecular action of insulin-sensitizing agents). Endokrynol Pol 56: 308-313, 2005 (In Polish).

19. Folsom AR, Anderson KE, Sweeney C and Jacobs DR Jr: Diabetes as a risk factor for death following endometrial cancer. Gynecol Oncol 94: 740-745, 2004.

20. Shoff SM and Newcomb PA: Diabetes, body size, and risk of endometrial cancer. Am J Epidemiol 148: 234-240, 1998.

21. Salazar-Martínez E, Lazcano-Ponce EC, Lira-Lira GG, Escudero-De los Rios P, Salmerón-Castro J, Larrea F and Hernández-Avila M: Case-control study of diabetes, obesity, physical activity and risk of endometrial cancer among Mexican women. Cancer Causes Control 11: 707-711, 2000.

22. Qu HQ, Li Q, Rentfro AR, Fisher-Hoch SP and McCormick JB The definition of insulin resistance using HOMA-IR for Americans of Mexican descent using machine learning. PLoS One 6: e21041, 2011

23. Habib SS, Eshki A, AlTassan B, Fatani D, Helmi H and AlSaif S: Relationship of serum novel adipokine chemerin levels with body composition, insulin resistance, dyslipidemia and diabesity in Saudi women. Eur Rev Med Pharmacol Sci 21: 1296-1302, 2017.
24. Zhang Y, Liu Z, Yu X, Zhang X, Lü S, Chen X and Lü B: The association between metabolic abnormality and endometrial cancer: A large case-control study in China. Gynecol Oncol 117: 41-46, 2010

25. Bray GA: The underlying basis for obesity: Relationship to cancer. J Nutr 132: 3451-3455, 2002

26. Akhmedkhanov A, Zeleniuch-Jacquotte A and Toniolo P: Role of exogenous and endogenous hormones in endometrial cancer: Review of the evidence and research perspectives. Ann N Y Acad Sci 943: 296-315, 2001.

27. Kaaks R, Lukanova A and Kurzer MS: Obesity, endogenous hormones, and endometrial cancer risk: A synthetic review. Cancer Epidemiol Biomarkers Prev 11: 1531-1543, 2002.

28. Osório-Costa F, Rocha GZ, Dias MM and Carvalheira JB: Epidemiological and molecular mechanisms aspects linking obesity and cancer. Arq Bras Endocrinol Metabol 53: 213-226, 2009.

29. Takemura Y, Ouchi N, Shibata R, Aprahamian T, Kirber MT, Summer RS, Kihara S and Walsh K: Adiponectin modulates inflammatory reactions via calreticulin receptor-dependent clearance of early apoptotic bodies. J Clin Invest 117: 375-386, 2007.

30. Higuchi A, Ohashi K, Shibata R, Sono-Ro manelli S, Walsh K and Ouchi N: Thiazolidinediones reduce pathological neovascularization in ischemic retina via an adiponectin-dependent mechanism. Arterioscler Thromb Vasc Biol 30: 46-53, 2010.

This work is licensed under a Creative Commons Attribution-NonCommercial-NoDerivatives 4.0 International (CC BY-NC-ND 4.0) License. 